\title{
Relationship of NF-kB expression with Astrocytoma Grade and Clinical Outcome
}

\author{
Khairul Muhajir*, Suzy Indharty, Andre Siahaan \\ Departement of Neurosurgery, Faculty of Medicine, University of Sumatera Utara/ H. \\ Adam Malik Hospital Medan, Indonesia
}

\begin{abstract}
In the diagnosis of glioblastoma multiform (GBM), there is often a disagreement between the WHO based astrocytoma grading and the patient's clinical outcome. NF-kB is assumed to be a good predictor of clinical outcome of astrocytoma. This study will determine the relationship of NF- $\mathrm{kB}$ expression with astrocytoma grade and clinical outcome. This analytical cross sectional study were conducted on 25 astrocytoma samples. Samples were analyzed by anatomical pathologists to determine WHO grading and immunohistochemically stained to analyze the expression of cytplasmic and nuclear NF- $\mathrm{kB}$. The clinical outcome was determined from the patient's survival at the end of therapy. The results showed that majority of astrocytoma patients were in grade I (36\%), had positive cytoplasmic NF- $\kappa \mathrm{B}$ expression $(88 \%)$, and negative nuclear NF- $\mathrm{KB}$ expression( $40 \%)$. A lower grade of astrocytoma and better outcomes were found in samples with positive cytoplasmic NF- $\mathrm{KB}$ expression or negative eotide NF- $\kappa \mathrm{B}$ expression. However, no cytoplasmic or nuclear NF- $\kappa \mathrm{B}$ expression relationship were found either with astrocytoma degree $(\mathrm{p}=0.543 ; \mathrm{p}=0.442)$ as well as clinical outcome of patients $(\mathrm{p}=0.472 ; \mathrm{p}=0.378)$. Thus, this study shows that NF- $\mathrm{KB}$ can not be used as a predictor of prognosis in patients with astrocytoma. Further research is needed to reassess this relationship with more focus on high grade astrocytoma.

Keywords : Glioblastoma multiform, NF- $\kappa B$, outcome.
\end{abstract}

\section{Introduction}

Glioblastoma multiform (GBM) is the most aggressive primary brain tumor in the adult nervous system with a poor prognosis. ${ }^{1}$ GBM is also the most common type of tumor in adults. ${ }^{2}$ The estimates of relative survival rates for glioblastoma are low which only about $4.5 \%$ of patients can survive 5 years after being diagnosed with this tumor. ${ }^{3}$

Gliomas are classified by the World Health Organization from grade I to IV based on the degree of differentiation, anaplasia, and aggressiveness. ${ }^{4}$ Grade III and IV are classifications for malignant gliomas, including anaplastic astrocytoma, anaplastic oligodendroglioma, oligoastrositoma anaplastic, and glioblastoma multiforme. ${ }^{5}$ Nevertheless, in patients with astroctoma, the severity by WHO grading (grading anatomical pathology) was often differ with the clinical phenotype of patients. Patients with grade 2 gliomas might exhibit poor clinical conditions, whereas patients with grade IV gliomas had small tumor sizes. 
Various pathogenesis pathways of molecular glioma affected the clinical presentation of gliomas such as the deletion of INK4A, EGFR amplification and mutation, loss of PTEN, and MDM2 amplification. ${ }^{6}$ Recent

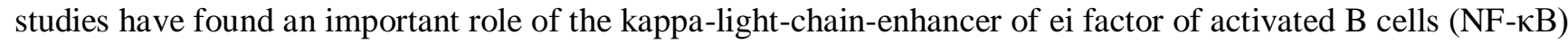
in GBM and implies NF- $\mathrm{KB}$ activation was a trigger for malignant phenotype which results in poor prognosis in GBM patients. ${ }^{7,8}$

$\mathrm{NF}-\mathrm{\kappa B}$ is a group of transcription factors that bind to the light chain of kappa immunoglobulin on active B cells. Structurally, NF- $\kappa B$ consists of homodimers and heterodimers of NF- $\kappa B 1, N F-\kappa B 2$, REL A, and c-

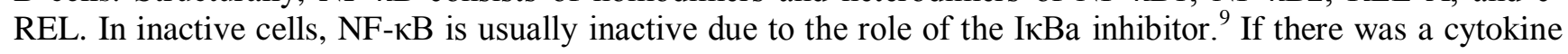
stimulus as well as DNA damage, IkBa will undergo phosphorylation, and eventually led to the release of the free NF- $\kappa B . N F-\kappa B$ then moved toward the eus. This mediator acted as a transcription factor of proinflammatory cytokines. ${ }^{10}$

Hayashi et al. (2001) and Korkopoulou et al. (2008) indicated that high NF-kB expression is associated with poor clinical and astrocytic degrees. ${ }^{11,12}$ This was also supported by many studies involving more research subjects. ${ }^{13,14,15}$ Therefore, it is important to know the relationship of cytoplasmic or nuclear NF- $\mathrm{kB}$ expression with astroctyoma grading based on WHO classification system and the clinical outcome in Indonesian patient. In the future, NF- $\mathrm{KB}$ can be promising to determine tumor aggressiveness more accurately, especially in cases with minimal correlation between clinical and histological parameters. This study will be conducted to determine the relationship of NF- $\mathrm{KB}$ expression in astrocytoma with WHO classification system and outcome.

\section{Methods}

\section{Subjects selection}

This study was a cross sectional analytic study that aimed to analyze the relationship between NF- $\kappa \mathrm{B}$ expression and astrocytoma degree based on WHO classification with clinical outcome of astrocytoma patients. This study has been approved by the Ethical Committee of the Faculty of Medicine of Sumatera Utara.A total of 25 consent astrocytoma patients who underwent surgery at the Department of Neurosurgery RSUP H Adam Malik Medan from January 2015 to September 2016 were included in the study. Patients with other malignancies become exclusion criteria.

\section{Parameters of study}

The primary parameters of the study were NF- $\mathrm{B}$ cytoplasmic expression, ear NF- $\kappa \mathrm{B}$ expression, astrocytoma grade based on WHO classification, and clinical outcomes of astrocytoma patients. The secondary parameters were the patients demographics.The specimens of tumor from surgery were analyzed in the anatomical pathology laboratory to confirm astrocytome diagnosis and a NF- $\mathrm{kB}$ expression. The medical records of patients were collected to determine patients demographic data.

\section{Analysis of NF-kB expression}

Paraffin blocks of astrocytoma patients were collected and confirmed by anatomical pathologists for further analysis. Cytoplasmic and eicNF- $\mathrm{KB}$ expression were analyzed immunohistochemically. The slides were washed with buffer for $5 \mathrm{~min}$, added $3 \% \mathrm{H} 2 \mathrm{O} 2$ for $15 \mathrm{~min}$, then blocked with $0.25 \%$ Triton protein after previously rinsed 3 times with buffer for each of $3 \mathrm{~min}$. The samples were then incubated using the primary antibody (NF-KB) for $60 \mathrm{~min}$ at room temperature and added SA-HRP (Strep-Avidin Horse Radis Peroxidase) for $10 \mathrm{~min}$ and diaminobenzidine for $10 \mathrm{~min}$. The stainings were done by counter staining method with Hematocycline Eosin for 1 minute. After rinsing with clean water and peroxide, the samples were mounted and checked by anatomical pathologist under a light microscope. Interpretation of NF-kB was categorized as negative or positive. On the other hand, the grade of astrocytoma based on the WHO classification were determined by processing the slides on eosin hematocycline. Histologic assessment was performed by anatomical pathologists under light microscope with low grade or high grade categorization.

\section{Clinical outcomes}

In patients, the clinical outcomes were assessed as the final outcome of therapy at home, whether alive or dead, judged by the dataof medical records. In addition, patient demographic characteristics such as age and gender were recorded as secondary data. 


\section{Data analysis}

The categorical variables are analyzed in terms of frequency and percentage presented in both tables and graphs. Descriptive analysis of numerical variables is done in the form of mean and standard deviation. Medical and demographic data were analyzed using computerized statistical Chi Square or Fischer's exact test with significance of $95 \%$ and power of $80 \%$.

\section{Results}

A total of 25 specimens of astrocytoma patients who had undergone tumor removal surgery at $\mathrm{H}$. Adam Malik General Hospital Medan was analyzed in this study. Majority of patients were male (60\%) with mean age 35.96 (SD 14.66) years (Table 1). Distribution of astrocytoma classification based on WHO grade showed that mostly subjects were grade I $(36,0 \%)$, followed bygrade II and grade IV astrocytoma $(24.0 \%)$ and grade 4 astrocytoma (16.0\%) (Table 2).

Table 1. Demographics of subjects

\begin{tabular}{|l|l|l|}
\hline Characteristics & n & \% \\
\hline Gender & & \\
\hline Male & 15 & 60 \\
\hline Female & 10 & 40 \\
\hline Age & & \\
\hline $0-9$ & 1 & 4 \\
\hline $10-19$ & 3 & 12 \\
\hline $20-29$ & 4 & 16 \\
\hline $30-39$ & 5 & 20 \\
\hline $40-49$ & 8 & 32 \\
\hline $50-59$ & 3 & 12 \\
\hline $60-69$ & 1 & 4 \\
\hline
\end{tabular}

Table 2. Distribution of astrocytoma grade based on WHO classification

\begin{tabular}{|l|l|l|}
\hline $\begin{array}{l}\text { Astrocytoma grade based on } \\
\text { WHO classification }\end{array}$ & $\mathbf{n}$ & \% \\
\hline I & 9 & 36 \\
\hline II & 6 & 24 \\
\hline III & 4 & 16 \\
\hline IV & 6 & 24 \\
\hline
\end{tabular}

Most of the samples in this study showed positive cytoplasmic NF-kB expression (88\%) but negative nuclear NF-kB expression(40\%) (Table 3). One subject who did not express both the nuclear and the cytoplasmic NF- $\kappa \mathrm{B}$ had low grade astrocytoma.

Table 3.Relationship of nuclear and cytoplasmic NF-кB expression with astrocytoma degree based on WHO classification

\begin{tabular}{|l|c|c|c|}
\hline \multirow{2}{*}{ NF-kB expression } & \multicolumn{2}{|c|}{ Astrocytoma grade based on WHO classification } & \multirow{2}{*}{ p value } \\
\cline { 2 - 3 } & High grade & \multicolumn{2}{|l}{} \\
\hline Cytoplasmic NF-kB expression grade & \multirow{2}{*}{} \\
\hline Positive & 8 & 14 & \multirow{2}{*}{0.442} \\
\hline Negative & 2 & 1 & \\
\hline Nuclear NF-kB expression & 5 & 5 & \\
\hline Positive & 5 & 10 & \\
\hline Negative & \multicolumn{3}{|l}{} \\
\hline
\end{tabular}


Table 4.Relationship of nuclear and cytoplasmic NF-KB expression with clinical outcome of astrocytoma patients

\begin{tabular}{|c|c|c|c|}
\hline \multirow{2}{*}{ NF-kB expression } & \multicolumn{2}{|c|}{ Clinical outcome } & \multirow{2}{*}{ value } \\
\cline { 2 - 3 } & Alive & Die & \multirow{2}{*}{} \\
\hline Cytoplasmic NF-kB expression & 5 & 0.180 \\
\hline Positive & 17 & 2 & \multirow{2}{*}{0.378} \\
\hline Negative & 1 & 4 & \\
\hline Nuclear NF-kB expression & 6 & 3 & \\
\hline Positive & 12 &
\end{tabular}

Most subjects with positive cytoplasmic NF-kB expression had low grade astrocytoma (64\%) and better outcomes with $77 \%$ of patients alive. Most subjects with negative NF-kB cytoplasmic expression had high grade astrocytoma (67\%) and worse outcome with $67 \%$ of patients died. However, there were no association between cytoplasmic NF- $\kappa B$ expression with astrocytoma degree $(p=0.543)$ and clinical outcome $(\mathrm{p}=0.180)$.

In the nuclear NF- $\mathrm{KB}$ expression analysis, most of the negative subjects had low grade classification $(67 \%)$ and better outcomes with $80 \%$ of patients remaining alive. Subjects with positive expression showed a balanced proportion of astrocytoma and clinical outcomes. The result of statistical test with Chi Square showed no difference between nuclear NF- $\mathrm{KB}$ expression both with astrocytoma grade $(\mathrm{p}=0.442)$ and clinical outcome $(\mathrm{p}=0.378)($ Table 4$)$.

Table 5 showed that there was a correlation between degree of astrocytoma based on WHO classification with clinical outcome $(p=0.001)$. The best outcomes of patients were highest in grade I astrocytoma (50\%) and worst outcomes were highest in grade IV astrocytoma (71\%).

Table 5. The relationship between astrocytoma grade based on WHO classification with clinical outcome of astrocytoma patients

\begin{tabular}{|c|c|c|c|}
\hline \multirow{2}{*}{$\begin{array}{l}\text { Astrocytoma grade } \\
\text { based on WHO } \\
\text { classification }\end{array}$} & Alive & Clinical outcome & \multirow{2}{*}{ Dalue } \\
\cline { 2 - 3 } & & & \multirow{2}{*}{0.001} \\
\hline I & 9 & 0 & \\
\hline II & 6 & 0 & \\
\hline III & 2 & 2 & \\
\hline IV & 1 & 5 & \\
\hline
\end{tabular}

\section{Discussion}

Astrocytoma is a neuroectodermal tumor derived from sustentacular neuroglia cells. Astrocytoma is classified into four grades where grade I and grade II are classified as low grade astrocytoma, while grade III and grade IV are classified into high grade glioma. ${ }^{16}$

Ostrom et al. (2013) showed that astrocytoma was more commonly found in men than women with a ratio of 1.5: 1 with an average age at diagnosis is about 40 years old. ${ }^{3}$ Similar to this study, astrocytoma was more commonly found in men with a ratio 3:2 with mean age of 35.96 years at most in the 40-49 year range $(32 \%)$ (Table 1). In women, astrocytomas were more common in menopausal age with a hypothesis that female hormonesbefor menopause had a protective effect on astrocytes. ${ }^{3}$

In this study, it was found that grade I astrocytoma based on WHO classification was the most common type of astrocytoma (36\%) followed by grade II and grade IV (4\%) and grade III (14\%)(Table 2). These results did not resemble previous studies where the highest incidence was found in grade II astrocytoma by $39.9 \%$ followed by grade IV astroscytoma of $36.2 \%$, grade astrositoma III of $14.3 \%$ and grade astrositoma I of $9.5 \%$. $^{3}$ 
Later, Verhaak et al integrated genomic analysis and categorized it in subsequent clinical-related subtypes of classical videlicet, neural, proneural and mesencalal. ${ }^{15}$

Modality of existing therapies were not curative. The biggest challenge was that astrocytoma molecular pathology was heterogeneous and aggressive with the difficulty of local control. Standard management includes radical surgery followed by radiotherapy or chemotherapy. ${ }^{16}$ In general, the combination of temozolamide and chemotherapy proves to be beneficial. There was a three-month increase in life expectancy $17 \%$. Less aggressive therapies, usually in the form of radiation or chemotherapy were sometimes recommended in the elderly or at high risk. In the group of patients over the age of 70 years, radiotherapy alone increased life expectancy compared to those who did not undergo radiotherapy. In general, mean life expectancy of GBM patients who undergo surgery alone was6.9 months. ${ }^{17}$

Many studies suggested that worse clinical outcomes were found in higher astrocytoma grades. ${ }^{4}$ This study showed that there was a relationship between astrocytoma grade based on WHO classification and clinical outcome $(\mathrm{p}=0.001)$. The best outcomes of patients were highest in grade I astrocytoma $(50 \%)$ and worst outcomes were in grade IV astrocytoma (71\%) (Table 5). Out of all 9 subjects with grade I astrocytoma and 6 subjectswith grade II astrocytoma were all alive. In grade III astrocytoma 2 subjects alive, and 2 subjects died. In patients with grade IV astrocytoma, there was 1 subject alive, and 5 subjects died. However, the poor clinical findings showed by the patient was often incompatible with astrocytoma grade, regardless of its relationship to the clinical outcome.

In this study, lower grade astrocytoma and better outcomes were observed in subjects with positive cytoplasmic NF- $\mathrm{kB}$ expression or negative nuclear NF-kB expression. However, no cytoplasmic or nuclear NF$\kappa \mathrm{B}$ expression relationship were found either with astrocytoma grade $(p=0.543 ; p=0.442)$ as well as clinical outcome of astrocytoma patients $(\mathrm{p}=0.472 ; \mathrm{p}=0.378$ ) (Table 3 , Table 4). Thus, this study showed that NF- $\mathrm{KB}$ can not be used as a predictor of prognosis in patients with astrocytoma.

These findings were very different from the others studies. Hayashi reported that the expression of nuclear NF- $\mathrm{KB}$ was closely related to the degree of astrocytoma. ${ }^{11}$ Contradiction was also shown Korkolopoulou et al. (2008) who found outcomes of patients with NF-kB expression but not with the WHO classification. However, researchers only took astrocytomas with high grade degree. ${ }^{12}$ Researchers assumed that differences in research methodology and small number of samples of low grade grade astrocytoma became a weakness of this study. However, further research is needed to reassess this case with more focus on high grade astrocytoma.

\section{Conclusions}

Neither cytoplasmic nor nuclear NF- $\mathrm{kB}$ expression were associated with astrocytoma grading and clinical outcome. So that NF-kB can not be used as a predictor of prognosis in patients with astrocytoma. Further research is needed to reassess this case with more focus on high grade astrocytoma. Markers of the EGFR pathway may also be considered as an external predictor option for astrocytoma patients.

\section{References}

1. D.A. Reardon, Oncologist, 11, 152 (2006); doi:10.1634/theoncologist.11-2-152.

2. H. Ohgaki and P. Kleihues, Clin. Cancer Res., 19, 764 (2013); doi:10.1158/1078-0432.CCR-12-3002.

3. Q.T. Ostrom, H. Gittleman, P. Farah, A. Ondracek, Y. Chen, Y. Wolinsky, N.E. Stroup, C. Kruchko and J.S. Barnholtz-Sloan, Neuro-oncol., 15, 1 (2013); doi:10.1093/neuonc/not151.

4. C.B. Colen and E. Allcut, Neurosurg. Clin. N. Am., 23, 507 (2012); doi:10.1016/j.nec.2012.04.010.

5. V.T. Puliyappadamba, K.J. Hatanpaa, S. Chakraborty and A.A. Habib, Mol. Cell. Oncol., 1, e963478 (2014); doi:10.4161/23723548.2014.963478.

6. G.P. Atkinson, S.E. Nozell and E.T.N. Benveniste, Expert Rev. Neurother., 10, 575 (2010); doi:10.1586/ern.10.21. 
7. F.B. Furnari, T. Fenton, R.M. Bachoo, A. Mukasa, J.M. Stommel, A. Stegh, W.C. Hahn, K.L. Ligon, D.N. Louis, C. Brennan, L. Chin, R.A. DePinho and W.K. Cavenee, Genes Dev., 21, 2683 (2007); doi:10.1101/gad.1596707.

8. S.J. Hentschel and F.F. Lang, Cancer J., 9, 113 (2003); doi:10.1097/00130404-200303000-00007.

9. Y.H. Kim and C.Y. Kim, Neurosurg. Clin. N. Am., 23, 199 (2012); doi:10.1016/j.nec.2012.01.010.

10. B.B. Taw, A.A. Gorgulho, M.T. Selch and A.A. De Salles, Neurosurg. Clin. N. Am., 23, 259 (2012); doi:10.1016/j.nec.2012.01.003.

11. S. Hayashi, M. Yamamoto, Y. Ueno, K. Ikeda, K. Ohshima, G.-I. Soma and T. Fukushima, Neurol. Med. Chir. (Tokyo), 41, 187 (2001); doi:10.2176/nmc.41.187.

12. P. Korkolopoulou, G. Levidou, A.A. Saetta, E. El-Habr, C. Eftichiadis, P. Demenagas, I. Thymara, K. Xiromeritis, E. Boviatsis, E. Thomas-Tsagli, I. Panayotidis and E. Patsouris, Hum. Pathol., 39, 1143 (2008); doi:10.1016/j.humpath.2008.01.020.

13. C.B. Colen and E. Allcut, Neurosurg. Clin. N. Am., 23, 507 (2012); doi:10.1016/j.nec.2012.04.010.

14. D.T. Nagasawa, F. Chow, A. Yew, W. Kim, N. Cremer and I. Yang, Neurosurg. Clin. N. Am., 23, 307 (2012); doi:10.1016/j.nec.2012.01.007.

15. R.G. Verhaak, K.A. Hoadley, E. Purdom, V. Wang, Y. Qi, M.D. Wilkerson, C.R. Miller, L. Ding, T. Golub, J.P. Mesirov, G. Alexe, M. Lawrence, M. O'Kelly, P. Tamayo, B.A. Weir, S. Gabriel, W. Winckler, S. Gupta, L. Jakkula, H.S. Feiler, J.G. Hodgson, C.D. James, J.N. Sarkaria, C. Brennan, A. Kahn, P.T. Spellman, R.K. Wilson, T.P. Speed, J.W. Gray, M. Meyerson, G. Getz, C.M. Perou and D.N. Hayes, Cancer Cell, 17, 98 (2010); doi:10.1016/j.ccr.2009.12.020.

16. A. Omuro, JAMA, 310, 1842 (2013); doi:10.1001/jama.2013.280319.

17. S. Ishkanian, N.J. Laperriere, W. Xu, B.-A. Millar, D. Payne, W. Mason and A. Sahgal, Cancer, 117, 4070 (2011); doi:10.1002/cncr.25988. 